\title{
Burnout. Soziales Leiden an Wachstum und Wettbewerb
}

In der steigenden Zahl von Burnout-Diagnosen manifestiert sich ein subjektives Leiden an einer Sozialordnung, die von hypertrophen Wettbewerben und ökonomischen Wachstumszwängen geprägt ist. Besonders vulnerabel sind Berufstätige dadurch, dass der hohe Einsatz, der von ihnen verlangt wird, vielfach auf den Wunsch trifft, Arbeit als bedeutenden Teil der eigenen Selbstverwirklichung betrachten zu können. Das soziale Leid der Erschöpfung hat inzwischen ein solches Ausmaß erreicht, dass die Kritik am Wachstumsregime zur Veränderung des kapitalistischen Geistes unter dem Vorzeichen der Nachhaltigkeit beitragen könnte.

SIGHARD NECKEL, GRETA WAGNER

\section{Einleitung}

Wer heute im Frankfurter Bankenviertel Sozialforschung betreibt und Gespräche mit Finanzexperten führt, stößt immer häufiger auf Berichte wie den eines 50-jährigen Investmentbankers, der Folgendes aus seiner Berufsbiografie erzählt:

„Nach sieben Jahren in der Zentrale war ich sehr müde und hatte Burnout. Da war ich so um die 40. Wir hatten früh Kinder gekriegt und natürlich geackert ohne Ende. Aber es war auch so, dass man sich dann erst so richtig selbst erlebt hat, beim Reisen zum Beispiel. Das ist natürlich toll, und dann die Goldkarte und die Platinkarte von Amex und so ein Zeugs. Dieses sich selbst erst spüren, wenn man so hyperaktiv ist. Sobald es dann still ist, wird es leer. Das ist bei vielen Investmentbankern so. Die brauchen diese Spannung des Deal-Machens - ,wir sind die Deal-Maker. Das führt zu immer mehr, mehr, mehr - bis man dann abstürzt. Also ich war total müde. Dann haben die Ärzte mir gesagt: ,Du musst sofort raus'. Hab’ dann eineinhalb Jahre gebraucht..."

In den 1970er Jahren, als der amerikanische Psychoanalytiker Herbert Freudenberger den Begriff „Burnout“ prägte, wurde das Ausgebranntsein vornehmlich in Sozialberufen und bei Lehrerinnen und Lehrern diagnostiziert. Rastloser beruflicher Einsatz, eine starke Identifikation mit der Arbeit und Frustration über die geringe Anerkennung des eigenen Tuns standen typischerweise Pate beim körperlichen und psychischen Zusammenbruch. Heute gilt Burnout als weitverbreitet in der gesamten Berufswelt - von Führungskräften der Wirtschaft über prominente Sportler, Autorinnen und Medienstars bis hin zu Wissenschaftlern, mittleren Angestellten und Hartz IV-Empfängern. Zwischen 2004 und 2012 sind, wie der Bundesverband der Betriebskrankenkassen berichtet, die Arbeitsunfähigkeitstage in Deutschland wegen Burnout um das 18-Fache gestiegen (vgl. BKK Bundesverband 2012, S. 43). Das Bundesministerium für Arbeit und Soziales schätzt, dass allein auf psychische Überlastung und Burnout der Ausfall von 54 Mio. Arbeitstagen zurückgeht, eine Zunahme gegenüber 2001 von $60 \%$ (vgl. Braun/Hillebrecht 2013, S. 16). Unter 40- bis 50-jährigen Berufstätigen mit einem hohen sozioökonomischen Status ist Burnout am meisten verbreitet. In unteren und mittleren Schichten hingegen, bei Jüngeren und stärker bei Frauen als bei Männern werden zunehmend Depressionen diagnostiziert. Nach aktuellen Studien des RobertKoch-Instituts leiden ca. 8 \% der Erwachsenen in Deutschland an einer diagnostizierten Depression (vgl. Hapke et al. 2012). Es lässt sich annehmen, dass sich dahinter nicht selten ähnliche Symptome wie jene verbergen, die bei Patienten aus höheren Sozialgruppen als Burnout festgestellt werden.

Dennoch stellt Burnout keine „Modekrankheit der Besserverdienenden“ dar, wie dies die Medien neuerdings gerne verbreiten, nachdem sie zuvor den publizistischen Wir- 
bel um Burnout selbst erst angeheizt haben. In den Zustand einer totalen Erschöpfung der körperlichen und psychischen Kräfte geraten auch Arbeitslose, alleinerziehende Mütter und gewöhnliche Arbeitnehmer. Führungskräfte mögen ein Burnout als „Verwundetenabzeichen der Leistungsgesellschaft“ (Schmidbauer 2012, S. 159) herzeigen können, das auf ihren besonderen Einsatz in der Berufswelt verweist, ohne sich dem Stigma einer Depression aussetzen zu müssen. Dieser symbolische Vorteil ändert jedoch nichts daran, dass für die Entstehung eines belastungsbedingten Zusammenbruchs ein zumeist länger anhaltender Leidensdruck verantwortlich ist.

Gleichwohl gehört Burnout nicht zu den medizinisch approbierten Krankheiten. Auch das Verzeichnis der Krankheiten und verwandter Gesundheitsprobleme der Weltgesundheitsorganisation von 2013 (ICD-10) kennt Burnout allein als „Problem der Lebensbewältigung“. Und in der Tat: Von einem akuten Burnout wird immer dann gesprochen, wenn sich eine emotionale Erschöpfung mit einer körperlichen Auszehrung verbindet und zu einem massiven Kollaps führt, der die Fortsetzung der bisherigen Lebensführung zumindest zeitweilig unmöglich macht. Chronischer Stress über längere Zeiträume hinweg, ein anhaltendes Gefühl der Überforderung oder Überlastung sowie Enttäuschungen im Beruf werden als Ursachen für Burnout benannt. Für die Umwelt macht sich dies etwa bemerkbar in einer starken Reizbarkeit der betroffenen Personen und einer „Depersonalisierung“ ihrer sozialen Kontakte, denen gegenüber sich Gleichgültigkeit, Distanz und Zynismus verbreitet.

In der Medizin mag die Frage, ob Burnout eine eigenständige Krankheit ist, die sich durch eindeutige Merkmale von körperlichen Erschöpfungszuständen, vom chronischen Müdigkeitssyndrom oder von Depressionen unterscheidet, von großer Bedeutung sein. Die Sozialforschung indes, die sich für Burnout als ein zeittypisches Phänomen der modernen Lebensführung und heutiger Arbeitswelten interessiert, braucht sich zwischen „krank“ oder "gesund“, „,normal“ oder „pathologisch“ nicht zu entscheiden. Wie Emotionen insgesamt ein Bindeglied zwischen Person und Gesellschaft sind, ein Vermittler zwischen dem Körper und dem Sozialen, so kommt auch der emotionalen Erschöpfung die soziologische Bedeutung zu, Auskunft über die Stellung des Einzelnen in seiner sozialen Umwelt zu geben, über Probleme und Konflikte zu informieren, die aus der jeweiligen Lebensführung entstehen.

Aus dem Blickwinkel der Soziologie stellt Burnout ein subjektives Leid dar, für das die medizinische Behandlungsdiagnose einer „Krankheit“ nicht entscheidend ist, da sich in ihm über individuelle Belastungen hinaus gesellschaftliche Probleme, insbesondere des modernen Berufslebens, dokumentieren. So sind sich medizinische, psychologische und sozialwissenschaftliche Experten auch weitgehend darin einig, dass Burnout ein meist arbeitsbedingtes Erschöpfungssyndrom darstellt, dessen Ursachen in den Belastungsfaktoren eines gesellschaftlichen Wandels zu suchen sind, der von dem Einzelnen in hohem Maße berufliche Einsatzbereitschaft, eine starke Identifikation mit der Arbeit, zeitliche Flexibilität, persönliche Eigenverantwortung und vermehrte Selbststeuerung bei der alltäglichen Lebensbewältigung einfordert.

Im Folgenden charakterisieren wir Burnout als eine Form sozialen Leidens am Wachstumsregime der Gegenwart: Ausgehend von der soziologischen Betrachtungsweise von subjektivem als sozialem Leid, wird die Wettbewerbsgesellschaft der Gegenwart als eine soziale Ordnung analysiert, die Leiden dadurch produziert, dass sie die Individuen in die permanente Sorge um ihre eigene Leistungsfähigkeit zwingt. Besonders vulnerabel werden sie dadurch, dass sie ihre immer größeren Anstrengungen in der Hoffnung auf eine berufliche Selbstverwirklichung erbringen, die nicht selten in Selbstausbeutung umschlägt. Erschöpfung vermag heute so umfassend die Krise des Wachstumsregimes zu beschreiben, dass Burnout aber auch als Beginn eines sozialen Wandels verstanden werden kann. Die Kritik an der Vernutzung von Subjektivität im Kapitalismus der Gegenwart kann die Herausbildung einer neuen Rechtfertigungsordnung bewirken, die nicht mehr nur individuelle Selbstverwirklichung, sondern auch einen schonenden Umgang mit subjektiven Ressourcen verspricht.

\section{Soziales Leiden und sozialer Wandel}

Psychisches Leiden als soziales Leid zu begreifen, gehört zum Grundverständnis der Soziologie. So zeigt Emile Durkheim (1983) in seiner Studie zum Selbstmord den Einfluss von sozialem Wandel, wirtschaftlichen Krisen und gemeinschaftlicher Integration auf die Selbstmordraten auf. Die scheinbar rein individuelle Handlung des Suizids, so weist Durkheim nach, wird durch soziale Faktoren beeinflusst: Die Anzahl der Suizide steigt in Zeiten sozialen Wandels, „,infolge schmerzhafter Krisen oder auch infolge günstiger aber allzu plötzlicher Wandlungen“ (ebd., S. 287). Zu jener Zeit, als Durkheim den Selbstmord als eine „soziale Tatsache“ untersuchte, öffnete sich der Blick für gesellschaftlich bedingtes Leiden insgesamt. Hierzu trug nicht wenig bei, dass in den letzten 20 Jahren des 19. Jahrhunderts in Europa und Nordamerika ein Erschöpfungssyndrom grassierte, das seinerzeit als Zeitkrankheit par excellence galt: die Neurasthenie. Das menschliche Nervensystem schien durch die modernen Lebensumstände, durch Fortschritte in Kommunikation und Transport und die damit einhergehende Beschleunigung überbeansprucht zu werden. So schrieb der deutsche Nervenarzt Wilhelm Erb: „Alles geht in Hast und Aufregung vor sich, die Nacht wird zum Reisen, der Tag für die Geschäfte benützt, selbst die Erholungsreisen werden zu Strapazen für das Nervensystem" (vgl. Kury 2013, S. 108). Die Lebensverhältnisse bürgerlicher Eliten hatten sich durch technische Innovationen in kurzer Zeit verändert und 
sollten zu Symptomen wie Kopfschmerzen, Reizbarkeit, Hoffnungslosigkeit, Schlaflosigkeit, Verlangen nach Drogen und Erschöpfung führen (vgl. ebd., S. 109). Auch Max Weber, der den gesellschaftlichen Wandel zwischen den Epochen soziologisch beschrieb, war Neurasthenie-Patient und litt jahrelang an dem nervösen Leiden (vgl. Kaube 2014, S. 116ff). Aber auch der Vorwurf, dass es sich bei der Neurasthenie um eine eingebildete Krankheit und eine Modediagnose handele, war Ende des 19. Jahrhunderts verbreitet. So schrieb 1888 der Psychiater Carl Pelman: „Jede Zeit hat, wie die sonstigen Moden, so auch ihre Modekrankheiten. Unter den modernsten dieser letzteren begegnen wir mit am häufigsten der Neurasthenie" (vgl. Kury 2013, S. 112). Der französische Soziologe Alain Ehrenberg hat die Neurasthenie sogar als die erste Modekrankheit überhaupt bezeichnet. Gleichwohl war sie der Ausgangspunkt für eine neue Aufmerksamkeit für das psychische Leiden an gesellschaftlichen Verhältnissen. Grundlegend für das moderne Verständnis von psychischem Leid wurde nun der „Begriff des Exogenen“: „Etwas, das von außen kommt, hat eine innere Wirkung, hat eine pathologische Reaktion der Person zur Folge“ (Ehrenberg 2013, S. 50).

Die soziologische Frage ist demzufolge, welchen Anteil bestimmte Zustände einer sozialen Ordnung daran haben, subjektives Leid zu erzeugen. In unserer Gegenwart dürfte die Studie Das Elend der Welt von Pierre Bourdieu dem gesellschaftlichen Leiden von Menschen die deutlichste Stimme verliehen haben (Bourdieu et al. 1997). Indem Bourdieu und sein Forschungsteam Personen von ihren Wohnverhältnissen und Arbeitsbedingungen, ihren Sorgen und Verletzungen erzählen lassen, bieten sie einen verstehenden Zugang zu menschlichen Nöten in Zeiten ökonomischer Transformationen und sich ändernder Sozialordnungen, die durch den Neoliberalismus bewirkt worden sind. Hieran anknüpfend ist unter dem Begriff „,social suffering" in den letzten Jahren eine interdisziplinäre Forschungsrichtung entstanden, die sich dem sozialen Leid in einer zweifachen Weise widmet: „Social suffering results from what political, economic, and institutional power does to people and, reciprocally, from how these forms of power themselves influence responses to social problems" (Kleinman et al. 1997, ix; vgl. auch Wilkinson 2005). Social suffering-Studien stellen gesellschaftlich geprägte Leidenserfahrungen in das Zentrum ihrer Untersuchungen - und die Arten und Weisen, in denen Personen ihre Leidenserfahrungen artikulieren.

Burnout als ein soziales Leiden, das wesentlich durch Arbeit hervorgerufen wird, gibt so Auskunft über die Belastungen, welche die Arbeitswelt der Gegenwart charakterisieren. Ihre Ursprünge hat die Burnout-Forschung in einem politisch-kulturellen Milieu, das die Art und Weise, in der heute gearbeitet wird, erstaunlich geprägt hat. Mitte der 1970er Jahre ist es der New Yorker Psychoanalytiker Herbert Freudenberger, der erstmals das Burnout-Syndrom beschreibt (Freudenberger 1974). Er entdeckte es im alternativen Helfermilieu von Sozialarbeitern, in dem Arbeit weit mehr darstellte als Quelle des Gelderwerbs zu sein. Als Psychoanalytiker, der sich in der Free-Clinic-Bewegung in Spanish Harlem und in der Arbeit mit Drogenabhängigen engagierte, beobachtete er bei sich und seinen Kollegen eine zunehmende emotionale Entleerung, Müdigkeit und zynische Einstellungen gegenüber den Klienten. Eine besonders große Gefahr des „Ausbrennens“ sah er in alternativen Institutionen, weil deren Mitarbeiter in ihrem hohen Engagement umso mehr mit den Zielen der Organisation identifiziert seien (Freudenberger 1975). Scheitere die Arbeit mit Klienten, entstehe aufgrund der Durchdringung von Privat- und Berufsleben und durch die Investition emotionaler Ressourcen das Gefühl, als ganze Person gescheitert zu sein (vgl. Pines et al. 1983, S 63).

Die psychologische Literatur, die in den letzten 40 Jahren über Burnout entstanden ist, zeichnet ein weitgehend übereinstimmendes Bild: Burnout folgt auf eine Phase immer größer werdender Anstrengungen, die vollbracht werden, um eigene oder fremde Ansprüche zu erfüllen (vgl. Schaufeli/Enzmann 1998). Von Burnout Betroffene versuchen Misserfolge mit verstärktem Einsatz zu kompensieren und geraten so in eine Spirale, bei der persönliche Ressourcen verausgabt werden, ohne je neue bilden zu können. Auch wenn Burnout gemeinhin als Leiden an schierer Überarbeitung gilt, so ist nicht die Überanstrengung allein verantwortlich für das „Ausbrennen“, sondern die Überanstrengung im Kontext bestimmter normativer Erwartungen. An Burnout Leidende haben hohe Ansprüche an ihre Arbeit ebenso wie an ihre eigene Arbeitskraft. Ihre berufliche Tätigkeit wollen sie mit Sinn erfüllt sehen, auch wenn sie dafür den Preis der Auszehrung zahlen müssen. Gerät die Berufsrealität in Konflikt mit ihren Intentionen und Wünschen, werden die Einsätze erhöht. Burnout tritt dann als selbstzerstörerischer Versuch auf, „unter Aufbietung aller Kräfte unrealistische Erwartungen zu verwirklichen“" (Freudenberger/Richelson 1983, S. 38).

\section{Wettbewerb und Wachstum}

Heute stellt Burnout nicht allein in sozialen Berufen, bei Lehrerinnen und Sozialarbeitern ein Problem dar. Unrealistische Erwartungen an die Belastbarkeit von Mitarbeitern entstammen längst nicht mehr dem Idealismus alternativer Milieus der 1970er Jahre, sondern sind in einer ökonomischen Kultur zur Regel geworden, die um jeden Preis auf permanente Leistungssteigerung setzt. Im Burnout-Syndrom artikuliert sich ein weit verbreitetes Unbehagen an den Arbeits- und Lebensbedingungen einer Wettbewerbsgesellschaft, das sich in bestimmten Fällen und Lebensphasen zu einer akuten Erschöpfungskrise steigert. Nicht einzelne Umstände beruflicher Belastung und Konkurrenz sind ursächlich hierfür, sondern das Zusammentreffen zahlreicher Stressfaktoren. In einer Arbeitswelt, die sich gegenüber 
dem Privatleben entgrenzt und alle auch subjektiven Energien und Motive einfordert, schlägt sich der zunehmende Wettbewerbsdruck in engmaschigen Leistungs- und Erfolgskontrollen nieder, die den Einzelnen dem Gefühl einer dauernden Bewährungsprobe aussetzen. Gepaart mit der Verdichtung und der Beschleunigung der alltäglichen Zeitorganisation sowie der Norm der permanenten Erreichbarkeit bricht durch die digitale Kommunikation der Beruf weitgehend in die Privatsphäre ein - Feierabend war gestern. Hohe Mobilitätsanforderungen bei gleichzeitiger Zunahme unsicherer und befristeter Beschäftigungsverhältnisse und die gestiegenen Ansprüche, die sowohl Arbeitgeber als auch Beschäftigte an die berufliche Tätigkeit richten, erhöhen den Druck, der auf dem alltäglich hohen Organisationsaufwand auch des Privat- und Familienlebens lastet. In der Arbeit und vielen anderen Lebensbereichen wird heute eine Art Selbstzuständigkeit erwartet, sodass man sich vom Verlauf des eigenen Berufswegs bis zum persönlichen Gesundheitsstatus und dem Schulerfolg der Kinder für alles selber verantwortlich fühlt. Dies hat vielfach zu einer „Verbetrieblichung" der alltäglichen Lebensführung geführt, der sich auf Dauer viele nicht mehr gewachsen sehen.

Der wesentlichste gesellschaftliche Faktor bei der „Verbetrieblichung " der alltäglichen Lebensführung ist die Ausbreitung von Wettbewerben, wie sich dies heute in Wirtschaft und Beruf, aber auch weit darüber hinaus im Sozialleben und in der populären Kultur niederschlägt. Wettbewerbe sind ein moderner Modus der Ressourcenallokation. Sie werden eingesetzt, um Ressourcen effektiv zu nutzen und zu verteilen, weshalb sie überall dort implementiert werden, wo das organisationale Ziel die Leistungssteigerung des Einzelnen ist. In Konkurrenz mit anderen zu stehen, soll Motivation und Einsatz beleben und den Erfolgswillen anspornen. Mit der Ausbreitung des Neoliberalismus seit den 1990er Jahren kam es zu einer zeitlichen und sachlichen Entgrenzung von Wettbewerben, sodass Wettbewerbe zunehmend die Sozialordnung als Ganze bestimmen. Einerseits wurden immer mehr Güter über Wettbewerbe verteilt, vormals öffentliche Institutionen dem Markt zugeführt. Andererseits wurden Wettbewerbe auch dort als Mittel zur Effektivitätssteigerung eingeführt, wo zuvor kein Markt existierte, wie etwa in Universitäten und öffentlichen Verwaltungen. Dies führt heute zu einer Entgrenzung von Wettbewerben, womit sich die zeitlichen Abstände, in denen Personen sich Wettbewerben ausgesetzt sehen, erheblich verkürzen. Der erreichte Status wird in immer kürzeren Abständen wieder zur Disposition gestellt und muss "performativ“ neu erkämpft werden (vgl. Rosa 2009, S. 662). Besonders bemerkbar macht sich dies in befristeten Arbeitsverhältnissen, die den Leistungsdruck auf die Mitarbeiter erhöhen und sie zwingen, ihren Wert für die Organisation immer wieder von Neuem beweisen zu müssen.

Wettbewerbe, die Ressourcen vermehren sollen, tragen so zu einer massiven Ressourcenvernichtung bei, weil sie die Individuen zwingen, am Ende nur noch in ihre eigene
Wettbewerbsfähigkeit zu investieren. Jeder Wettbewerb produziert Gewinner und Verlierer. Besonders viele Verlierer werden auf „winner-take-all“-Märkten produziert, bei denen die Ersten weit mehr erhalten als alle Nächstplazierten zusammen. In dem Maße, wie in Zeiten vertiefter sozialer Ungleichheit solche „alles-für-einen“-Strukturen zunehmend die Verteilungsordnungen charakterisieren (vgl. Neckel 2014), verschwenden immer mehr Konkurrenten ihre Ressourcen an einen destruktiv gewordenen Wettbewerbsmodus. Ein Beispiel hierfür ist die Konkurrenz um Drittmittel an Hochschulen und Universitäten, die zu unzähligen aufwendigen Anträgen für Forschungsarbeiten führt, die nie durchgeführt werden. In Wirtschaftsunternehmen wiederum wird vielfach nicht mehr der betriebene Aufwand einer Arbeitsleistung gratifiziert, der etwa in Arbeitsstunden messbar ist, sondern der finanzielle Erfolg, den die Arbeitsleistung im Wettbewerb mit anderen erzielt (vgl. Neckel 2008). Wie lange man arbeiten muss, um diesen Erfolg zu erlangen, liegt in der eigenen Hand. Dies bedeutet nicht nur, dass Wettbewerbe viele Verlierer produzieren, deren Anstrengungen nicht anerkannt werden, sondern auch, dass eine Arbeitskraft nie weiß, wann genug gearbeitet wurde und ob die investierte Zeit bereits ausreicht, um besser zu sein als die Konkurrenz. Dies ist einer der Hintergründe dafür, dass Burnout vielfach aus einem Zusammenspiel von Überanstrengung und Gratifikationskrise entsteht, also als Ergebnis eines dauerhaften Stresses, der zu keiner Belohnung führt. Wenn Wettbewerbe sich zu einer dominanten Sozialform der Gegenwart verwandeln und es alltäglich wird, sich im Modus der Konkurrenz zu begegnen, verschleißen Wettbewerbe jene subjektiven Kräfte, die sie vorgeben zu steigern.

\section{Selbstverwirklichung und Vulnerabilität}

Aber nicht nur die Entgrenzung von Wettbewerben, die in alle Poren des Soziallebens eindringen, macht Menschen anfällig für die völlige Erschöpfung. Hinzu tritt als „subjektiver Faktor“ der Wunsch vieler danach, dass die Arbeit mehr sein möge als bloßer Lebensunterhalt. Ebenso wie die Burnout-gefährdeten Helfer aus den 1970er Jahren, die danach strebten, mit ihrem Einsatz zu einer besseren Welt beizutragen und die dabei in eine Spirale immer größerer Anstrengungen und Enttäuschungen gerieten, macht heute der Wunsch nach beruflicher Selbstverwirklichung anfällig dafür, persönlich zu viel in die Arbeit zu investieren.

Die Burnout-Forschung der 1970er und 80er Jahre sah aufgeblähte Bürokratien, starre Hierarchien, stumpfe Routinen und kleinteilige Kontrollen als Ursachen für das „Ausbrennen“. Burnout werde begünstigt durch „eingefleischte Gewohnheiten“, „unterdrückte Phantasie“ und „me- 
chanisches Reagieren auf die Umgebung", wie es in einem zeittypischen Sachbuchtitel zu Burnout heißt (Bronsberg/ Vestlund 1988). Mit Luc Boltanski und Ėve Chiapello (2006) lässt sich eine solche Art von Kritik als „Künstlerkritik“ identifizieren: Sie richtet sich gegen bürokratische und tayloristische Formen der Arbeitsorganisation und erhebt die Forderungen nach Autonomie und Selbstverwirklichung. Ihr Gegenstand ist nicht zuvorderst die Ungerechtigkeit der kapitalistischen Wirtschaftsorganisation, sondern die Unfreiheit und Inauthentizität, die der Kapitalismus hervorbringt, und seine mangelnden Möglichkeiten zur persönlichen Selbstentfaltung. Heutige Arbeitsformen haben die Forderungen nach Flexibilisierung, Entbürokratisierung und Subjektivierung der Arbeit vielfach aufgenommen und stellen dies als Zugewinn an Authentizität und Autonomie dar. Insofern in Unternehmen „die individuellen Selbstentwürfe und Selbstverwirklichungsansprüche aktiviert und gezielt zur Prozessoptimierung nutzbar gemacht" (Bröckling 2000, S. 142) werden, ist aus der Forderung nach beruflicher Selbstverwirklichung eine Anforderung an moderne Arbeitnehmer geworden (vgl. Honneth 2002).

Die eigene Arbeit als Ergebnis eines intrinsischen Interesses darzustellen, gehört zu den Zumutungen der Gegenwart. Aktivität, Selbstveränderung und Engagement sind die neuen Werte am Arbeitsplatz. Der Wunsch, sich persönliche Erfolge durch hohe Motivation und pausenlosen Einsatz zu sichern, lässt Arbeitnehmer in eine gefährliche Verknüpfung von Selbstverwirklichung und Selbstausbeutung geraten. Die schwedische Soziologin Elin Thunman (2013) hat Burnout-Patienten interviewt, die mindestens ein halbes Jahr krank geschrieben waren. Sie beschreibt die große Bedeutung, welche die Arbeit für ihre Interviewpartnerinnen hatte. Alle gaben an, ihrem Beruf mit Leidenschaft nachzugehen und emotional stark involviert zu sein. Eine 43-jährige Vorschullehrerin zitiert Thunman mit den Worten: „Ich habe das Bedürfnis, die Initiative zu übernehmen. Ich brauche ein Ventil für meine Kreativität. Wenn es stagniert, bin ich gelangweilt. Und ich fühle mich in der Berufsrolle eingeengt, wenn ich nicht so frei arbeiten kann. Ich brauche diesen Flow“ (ebd., S. 64). Eine 36-jährige Hotelangestellte sagt über die Zeit, bevor sie Burnout bekam: „Je mehr ich tue und je kompetenter ich bin, umso besser bin ich als Person. [...] Und dann denkt man natürlich, wenn ich bei der Arbeit gut bin, dann ist das meine Identität als Mensch. Ich war meine Arbeit“ (ebd., S. 68f.). Die Befragten berichteten aber auch, dass ihre Arbeitgeber zwar eine hohe Identifikation mit dem Job einforderten, gleichzeitig aber nicht die Ressourcen zur Verfügung stellten, um die beruflichen Aufgaben zur eigenen Zufriedenheit zu erfüllen. „Alle waren bestrebt, sich selbst und ihren Werten bei der Arbeit treu zu bleiben, sie hatten die Bedingungen, unter denen sie ihre Tätigkeiten verrichteten, jedoch nicht wirklich in der Hand“ (ebd., S. 76). Die in Aussicht gestellte Selbstverwirklichung wurde so zum Köder für eine Falle: die Identifikation mit der Arbeit trieb die späteren Burnout-Patienten, je unzulänglicher die Arbeitsbedingungen waren, in immer größeres Engagement, was schließlich zum Erschöpfungszusammenbruch führte.

Die „Ausgebrannten“, mit denen die Soziologin Thunman sprach, haben viel gemeinsam mit den Sozialarbeiterinnen und Lehrern der 1970er und 1980er Jahre, auch wenn sie in ganz anderen Bereichen tätig sind. Der bedeutende Unterschied zwischen den Helferberufen vor 40 Jahren und den Angestellten der Gegenwart besteht jedoch darin, dass das hohe Ausmaß an Identifikation mit der Arbeit, das bei den Sozialberufen noch als „Helfersyndrom“ verspottet wurde, nunmehr gefordert wird. Die Angst, ersetzt zu werden, motiviert dazu, Überstunden zu machen. Eine Anfrage im Bundestag ergab, dass die Zahl der Beschäftigten, die abends arbeiten, innerhalb von zehn Jahren um $46 \%$ angestiegen ist, und die Anzahl der Beschäftigten mit überlangen Arbeitszeiten in den vergangenen zehn Jahren um 23 \% zugenommen hat (vgl. Krellmann 2013). Gerade posttayloristische Managementformen, bei denen Mitarbeitern freigestellt wird, auf welche Weise sie vereinbarte Ziele erreichen, führen dazu, dass die Arbeitszeit verlängert und verdichtet wird: „Was den Beschäftigten als ,Freiheit` versprochen wird, erweist sich bei genauerem Hinsehen als höchst ambivalente Selbstverantwortlichkeit“ (Haubl/Voß 2009, S. 7).

\section{Endliche Ressourcen und Postwachstum}

Unter dem Begriff Burnout ist in den letzten Jahren ein Leiden an der Arbeit in der Wettbewerbsgesellschaft entstanden, das weitgehend frei ist vom Stigma individuellen Versagens. Der Begriff des „Ausbrennens“ birgt die Vorstellung, vorher für die Arbeit gebrannt zu haben und attestiert den Betroffenen trotz ihres Scheiterns einen gesellschaftlich anerkannten Einsatz. In Talkshows, Sachbüchern und den Titelgeschichten zahlloser Magazine wurde durch den Begriff Burnout ein medialer Raum geschaffen, Überanstrengung und Überforderung zum öffentlichen Thema zu machen. Der Wandel der Arbeitswelt und die daraus entstehenden psychischen Kosten, welche die Arbeitssoziologie teilweise bereits seit Jahrzehnten beschrieben hatte, fanden Eingang in gesellschaftliche Debatten. Burnout wurde auf diese Weise zu einem Verdichtungssymbol, mit dessen Hilfe Kritik an steigendem Leistungsdruck und den unmäßigen Anforderungen in der modernen Arbeitswelt artikuliert werden kann. „Burnout bezeichnet niemals bloß individuelles Leiden, sondern stets auch gesellschaftliche Pathologie; der Burnout-Patient ist nicht nur medizinischer Fall, sondern vor allem Sozialfigur“" (Bröckling 2013, S. 179).

Trotzdem ist der normative Status von Burnout in gewisser Weise prekär, weil sich mit dem Erschöpfungssyndrom recht unterschiedliche Botschaften verbinden lassen. Vielen gelten die Zumutungen der gegenwärtigen Arbeitswelt als unvermeidbar in einer globalisierten Welt, in der 
Unternehmen um Konkurrenzfähigkeit kämpfen, weshalb sie von ihren Mitarbeitern ein hohes Maß an Wettbewerbsorientierung verlangen. Seit der Finanzkrise scheint es überdies ein Grund zur Dankbarkeit geworden sein, überhaupt arbeiten zu dürfen. Während die südeuropäischen Nachbarländer von Rezession gebeutelt sind und sich die Krise dort in Arbeits- und Perspektivlosigkeit, Armut und mangelnder Krankenversorgung ausdrückt, ist Deutschlands Wirtschaft Krisenprofiteur und wächst. Vor diesem Hintergrund steht das Leiden an der Arbeitswelt unter einem besonderen Legitimationsdruck, der nicht selten in den Vorwurf umschlägt, von Burnout Betroffene würden lamentieren und Anerkennung für ihre Wohlstandsprobleme einfordern. In einer Gesellschaft, die Fortschritt und Wohlergehen mit wirtschaftlichem Wachstum in eins setzt, ist das Leiden derer, denen alles über den Kopf wächst, nicht selten das Ziel von Häme und Spott.

Die Burnout-Ratgeber indes sind sich einig: Was das Individuum tun kann, um Burnout zu vermeiden, ist vor allem, einen schonenden Umgang mit subjektiven Ressourcen auszubilden. Schon ein Blick auf die Buchcover des Genres zeigt die Omnipräsenz der Erschöpfungsmetapher: Männer im Anzug, die ihre Kräfte in rasenden Hamsterrädern vergeuden; leere Akkus, die nicht früh genug wieder aufgeladen wurden. Managern wird in solchen Ratgebern empfohlen, die Ressourcen ihrer Mitarbeiter nicht zu verschwenden, um ein „organizational burnout “ zu verhindern (Greve 2012). Den von Burnout betroffenen Angestellten hingegen empfehlen die Ratgeber eine Bewusstwerdung darüber, wo die Quellen ihrer subjektiven Fähigkeiten liegen und wie sie ihre persönliche Lebensführung als Ausgleich zur Arbeit nutzen können. Sport, gegenseitige Wertschätzung, Erholungs- und Distanzierungsfähigkeit von der Arbeit werden als Ressourcen begriffen, die Burnout unwahrscheinlicher machen und die den Betreffenden helfen, chronischen Stress länger zu ertragen (vgl. Lanz 2010, S. 19). Auch Zeitmanagement und Work-Life-Balance gelten als geeignete Selbsttechniken, um ein „Ausbrennen“ zu vermeiden (Weimer/Pöll 2012; Scharnhorst 2012; Bergner 2010). Die heutige Wettbewerbsgesellschaft sieht jedoch einen schonenden Umgang mit subjektiven Ressourcen kaum vor. So sehr sich die zeitlichen Abstände verkürzen, in denen Positionen in Wettbewerben errungen und wieder verteidigt werden müssen, so wenig ermöglicht die damit entstehende Dynamisierung des Statuskampfes eine langfristige Einteilung der körperlichen und psychischen Kräfte. Endet diese Dynamisierung im Kollaps, so tritt zunächst Stagnation ein. Ausgebrannte sind gelähmt, passiv, emotionslos, sie haben keine Ressourcen mehr, die sie einbringen, geschweige in Wert setzen könnten - da die Wettbewerbsgesellschaft sie zwang, ihr gesamtes Potenzial restlos zu verbrauchen.

Trotz dieser Stagnation kann die Erschöpfung aber auch als Beginn eines Wandels verstanden werden, der Personen wie Organisationen umfasst. Der eingangs zitierte Investmentbanker beschreibt seinen psychischen Zusammenbruch als Endpunkt einer fortwährenden Steigerung der eigenen Aktivität. Er habe „geackert ohne Ende“ und sich selbst erst dann gespürt, wenn er „hyperaktiv“ gewesen sei. Mit den Worten „immer mehr, mehr, mehr - bis man dann abstürzt" illustriert er seinen psychischen Kollaps als Effekt des scheinbar grenzenlosen Wachstums seiner persönlichen Anstrengungen. Die hohe Frequenz seiner Handlungseinheiten und sein Streben nach Statuszuwachs führen ihn schließlich in den Zustand einer umfassenden Erschöpfung. Damit ist nicht gesagt, dass der erschöpfte Investmentbanker beginnen würde, die Wachstumslogik als solche infrage zu stellen. Doch ist er aus der Burnout-Klinik mit Techniken nachhaltigen Ressourcenmanagements zurückgekehrt, die ihn lehren, die Identifikation mit der Arbeit zu lockern und das Arbeitspensum zu senken.

Oft steht an der Quelle der Erschöpfung auch nicht die Forderung von Vorgesetzten, Überstunden zu machen, sondern der Wunsch von Angestellten selbst, ihre Arbeit so zu verrichten, dass sie mit den Ergebnissen persönlich zufrieden sind und ihre berufliche Tätigkeit als bedeutenden Teil ihrer Selbstverwirklichung betrachten können. In flexibilisierten Arbeitsstrukturen scheint zudem jeder frei darin zu sein, selbst über den Aufwand zu entscheiden, den er oder sie investieren möchte. All dies mündet geradewegs in den paradoxalen Umschlag von Selbstverwirklichung in Selbstausbeutung.

Im Zeichen derartiger Entwicklungen verändern sich gegenwärtig auch die Rechtfertigungsbedingungen des heutigen Kapitalismus. Noch Ende der 1990er Jahre machten Boltanski und Chiapello eine Rechtfertigungsordnung aus, in der flexible Netzwerkstrukturen die Teilnahme an der kapitalistischen Konkurrenz attraktiv erschienen ließen. Heute indes ist fraglich, ob diese Teilnahme nicht bereits anders gerechtfertigt wird, ob das Versprechen auf persönliche Freiheit und Selbstverwirklichung noch genug Anziehungskraft besitzt in einer Zeit, die sich selbst vorrangig im Begriff der Erschöpfung versteht. Erschöpfung ist heute ein Zustand, der sich nicht nur in der psychischen Verfassung von Arbeitspersonen, in ansteigenden Diagnosen von Burnout, Depression und chronischem Müdigkeitssyndrom manifestiert. Auch die wirtschaftliche Überschuldung, die Vernutzung endlicher Ressourcen und die Verknappung von Fürsorge und sozialer Sicherheit sind Symptome von Erschöpfungskrisen des Wachstumsregimes. Die öffentliche Kritik, die diese Krisen finden, könnte den Beginn einer neuen Rechtfertigungsordnung des Kapitalismus bilden, in deren Zentrum der Begriff der Nachhaltigkeit steht. Schon finden wir erste Anzeichen dafür in Unternehmen, die gelernt haben, die knappe Ressource qualifizierter Arbeitskräfte nachhaltiger zu bewirtschaften und in denen Maßnahmen für die Förderung der psychischen Gesundheit ergriffen werden. Die Kritik an dem verbreiteten sozialen Leiden am Wachstumsregime vermag so möglicherweise, zu einer Erneuerung des Kapitalismus unter dem Vorzeichen der „sustainability“ beizutragen. Es sind solche Innovationen des kapitalistischen Geistes, die ihn vor seiner eigenen Erschöpfung bewahren. 


\section{LITERATUR}

Bergner, T. M. H. (2010): Burnout-Prävention: Sich selbst helfen. Das 12-Stufen Programm, Stuttgart

BKK Bundesverband (2012): Seelische Gesundheit, BKK-Faktenspiegel 5/2012, Essen

Boltanski, L./Chiapello, E. (2006): Der neue Geist des Kapitalismus, Konstanz Bourdieu, P. et al. (1997): Das Elend der Welt. Zeugnisse und Diagnosen alltäglichen Leidens an der Gesellschaft, Konstanz

Braun, E./Hillebrecht, S. (2013): Betriebliche Wahrnehmung des Burnouts, in Der Betriebswirt. Management in Wissenschaft und Praxis 54 (3), S. 16-22 Bröckling, U. (2000): Totale Mobilmachung. Menschenführung im Qualitätsund Selbstmanagement, in: Bröckling, U./Krasmann, S./Lemke, T. (Hrsg.): Gouvernementalität der Gegenwart. Studien zur Ökonomisierung des Sozialen Frankfurt a. M., S. 131-167

Bröckling, U. (2013): Der Mensch als Akku, die Welt als Hamsterrad. Konturen einer Zeitkrankheit, in: Neckel, S.Magner, G. (Hrsg.): Leistung und Erschöpfung, a. a. O., S. $179-200$

Bronsberg, B./Vestlund, N. (1988): Ausgebrannt. Die egoistische Aufopferung, München

Durkheim, E. (1983): Der Selbstmord, Frankfurt a. M.

Ehrenberg, A. (2013): Das erschöpfte Selbst. Depression und Gesellschaft in der Gegenwart, Berlin

Freudenberger, H. J. (1974): Staff burn-out, in: Journal of Social Issues 30 (1) S. $159-165$

Freudenberger, H. J. (1975): The staff burn-out syndrome in alternative institutions in: Psychotherapy. Theory, Research and Practice 12 (1), S. 73-82

Freudenberger, H. J./Richelson, G. (1983): Mit dem Erfolg leben, München

Greve, G. (2012): Organizational Burnout. Das versteckte Phänomen ausgebrannter Organisationen, Wiesbaden

Hapke, U./Maske, U./Busch, M./Schlack, R./Scheidt-Nave, C. (2012): Stress, Schlafstörungen, Depressionen und Burnout: Wie belastet sind wir?, in: Bundesgesundheitsblatt-Gesundheitsforschung-Gesundheitsschutz 2012, Berlin, S. 987-988, www.rki.de/DE/Content/Gesundheitsmonitoring/Studien/Degs/ BGBL_2012_55_BM_Kurth.pdf?_blob=publicationFile

Haubl, R./Voß, G. G. (2009): Psychosoziale Kosten turbulenter Veränderungen. Arbeit und Leben in Organisationen 2008, in: Positionen. Beiträge zur Beratung in der Arbeitswelt 1/2009, S. 2-8

Honneth, A. (2002): Organisierte Selbstverwirklichung. Paradoxien der Individualisierung, in: Honneth, A. (Hrsg.): Befreiung aus der Mündigkeit. Paradoxien des gegenwärtigen Kapitalismus, Frankfurt a. M./New York, S. $141-158$

Kaube, J. (2014): Max Weber. Ein Leben zwischen den Epochen, Berlin Kleinman, A./Das, V./Lock, M. (Hrsg.) (1997): Social suffering, Berkeley Krellmann, J. (2013): Atypische Arbeitszeiten dehnen sich aus, psychische Belastungen nehmen weiter zu, http://www.linksfraktion.de/nachrichten/ atypische-arbeitszeiten-dehnen-sich-aus-psychische-belastungen-nehmenweiter

Kury, P. (2013): Von der Neurasthenie zum Burnout - eine kurze Geschichte von Belastung und Anpassung, in: Neckel, S./Wagner, G. (Hrsg.): Leistung und Erschöpfung, a.a.O., S. 107-128

Lanz, C. (2010): Burnout aus ressourcenorientierter Sicht im Geschlechterver gleich. Eine Untersuchung im Spitzenmanagement in Wirtschaft und Verwaltung, Wiesbaden

Neckel, S. (2008): Flucht nach vorn. Die Erfolgskultur der Marktgesellschaft, Frankfurt a. M./New York

Neckel, S. (2014): Oligarchische Ungleichheit. Winner-take-all-Mechanismen in der (obersten) Oberschicht, in: WestEnd, Neue Zeitschrift für Sozialforschung 11 (2) (im Erscheinen)

Neckel, S./Wagner, G. (Hrsg.) (2013): Leistung und Erschöpfung. Burnout in der Wettbewerbsgesellschaft, Berlin

Pines, A./Aronson, E./Kafry, D. (1983): Ausgebrannt. Vom Überdruß zur Selbstentfaltung, Stuttgart

Rosa, H. (2009): Von der stabilen Position zur dynamischen Performanz. Beschleunigung und Anerkennung in der Spätmoderne, in: Forst, R./Hartmann, M./Jaeggi, R./Saar, M. (Hrsg.): Sozialphilosophie und Kritik, Frankfurt a. M., S. $655-671$

Scharnhorst, J. (2012): Burnout. Präventionsstrategie und Handlungsoptionen für Unternehmen, Freiburg

Schaufeli, W. B./Enzmann, D. (1998): The burnout companion to study and practice. A critical analysis, London
Schmidbauer, W. (2012): Mehr Hofnarr als Hofrat. Über die Krisen der Psychotherapie, in: Nassehi, A. (Hrsg.): Krisen lieben, Kursbuch 170, Hamburg, S. $150-173$

Thunman, E. (2013): Burnout als sozialpathologisches Phänomen der Selbstverwirklichung, in: Neckel, S./Wagner, G. (Hrsg.): Leistung und Erschöpfung, a.a.O., S. $58-85$

Weimer, S./Pöll, M. (2012): Burnout. Ein Behandlungsmanual. Baukastenmodul für Einzeltherapie und Gruppen, Klinik und Praxis, Stuttgart

Wilkinson, I. (2005): Suffering. A sociological introduction, Cambridge

\section{AUTOREN}

SIGHARD NECKEL, Dr. habil., ist Professor für Soziologie und Soziale Ungleichheit am Fachbereich Gesellschaftswissenschaften der Goethe-Universität Frankfurt a. M. sowie Mitglied des Kollegiums des Instituts für Sozialforschung. Arbeitsschwerpunkte: Ungleichheitsforschung, Wirtschaftssoziologie, Gesellschaftsdiagnose, Emotionssoziologie.

neckel@soz.uni-frankfurt.de

GRETA WAGNER, Dipl.-Soz., ist Wissenschaftliche Mitarbeiterin am Lehrstuhl fü Soziologie und Soziale Ungleichheit im Fachbereich Gesellschaftswissenschaften der Goethe-Universität Frankfurt a. M. Arbeitsschwerpunkte: Medizin- und Körpersoziologie, Emotionssoziologie, Soziologien des Selbst.

greta.wagner@soz.uni-frankfurt.de 